\title{
Genetic Analysis and Age Determination of Chinook Salmon in the California Central Valley
}

\author{
J.J. Youngblom ${ }^{*}$, J. Mullins ${ }^{*}$, J.H. Youngblom ${ }^{*}$, and T. Heyne ${ }^{* *}$ \\ *California State University-Stanislaus, Biology Dept., Turlock, CA 95382 \\ **California Department of Fish and Game, Le Grange, CA 95333
}

Salmon populations around the world have suffered from habitat loss and overfishing, and in the Pacific Northwest there are 26 subspecies of salmon classified as endangered. The future survival of the salmon populations is directly related to the management strategies of these populations. Two critical components of salmon management are age determination of the adults returning to spawn, and genetic analysis of the populations. The purpose of this study is to analyze the genetic structure of the salmon populations of three San Joaquin River tributaries in the California central valley using DNA isolated from salmon scales, and to develop a more efficient and accurate method for age determination using salmon scales, and eventually otoliths, analyzed with a confocal laser scanning microscope.

Nuclear microsatellite DNA analysis is being conducted on the scales of the Chinook salmon isolated from three San Joaquin River tributaries. Currently, we have examined four loci determined by outside investigators to amplify polymorphic microsatellites in other Chinook salmon. Tissue from one and two scales was used, and the DNA was extracted with 5\% chelex solution. The DNA was amplified by the polymerase chain reaction (PCR) using primers labeled with a Hex fluorescence tag on one end. The PCR products were submitted to the DNA Sequencing Facility at Iowa State University for genetic haplotyping. Age determination using salmon scales are being conducted with a confocal laser scanning microscope. The autofluorescence of the scales allow automation of annuli ring counts using quantitative analysis software available on the Leica confocal system.

Comparative analysis of the genotype results from three microsatellite loci indicate a significant heterogeneity among the salmon samples tested. The number of alleles and range of base pairs for each of the 3 successfully amplified loci are as follows: Ots-3: 3 salmon $\rightarrow 4$ alleles (size range83-103 b.p.); Ots-9: 4 salmon $\rightarrow 2$ alleles (size range- 122-144 b.p.); Ots 10: 3 salmon $\rightarrow 4$ alleles (size range- 180-204 b.p.). One locus did not amplify. In contrast to the traditional method of annuli counting for age determination using microfiche, the confocal laser scanning microscope provided the distinct advantage of allowing automation of annuli counting and easy archiving of the images for comparative analysis of the scales.

DNA from a single fish scale appears to be sufficient to conduct genetic analysis of salmon populations. This capability will allow us to obtain DNA from a rare archived collection of fish scales that date back to the mid 1920's. These samples will provide a unique opportunity to compare the genetics of the present day fish to those that existed many generations ago. This multigenerational historical perspective could provide valuable information regarding how much genetic variation has passed from one generation to the next. The ease and efficiency of using the confocal laser scanning microscope for age determination of salmon scales and eventually otoliths should help accelerate the rate of collecting this vital data information. 


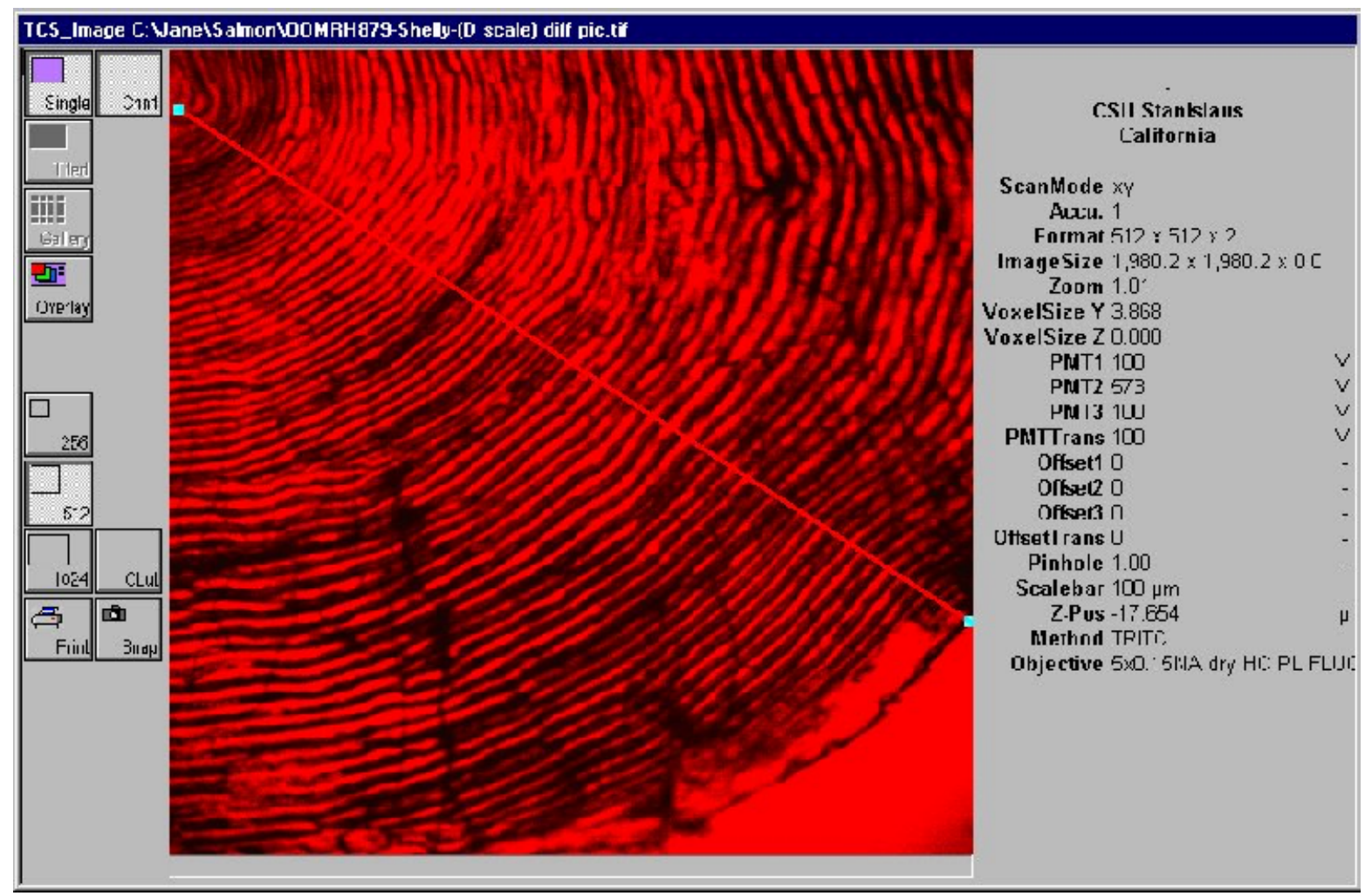

Figure 1. Confocal laser scanning microscope image of a salmon scale using a krypton laser

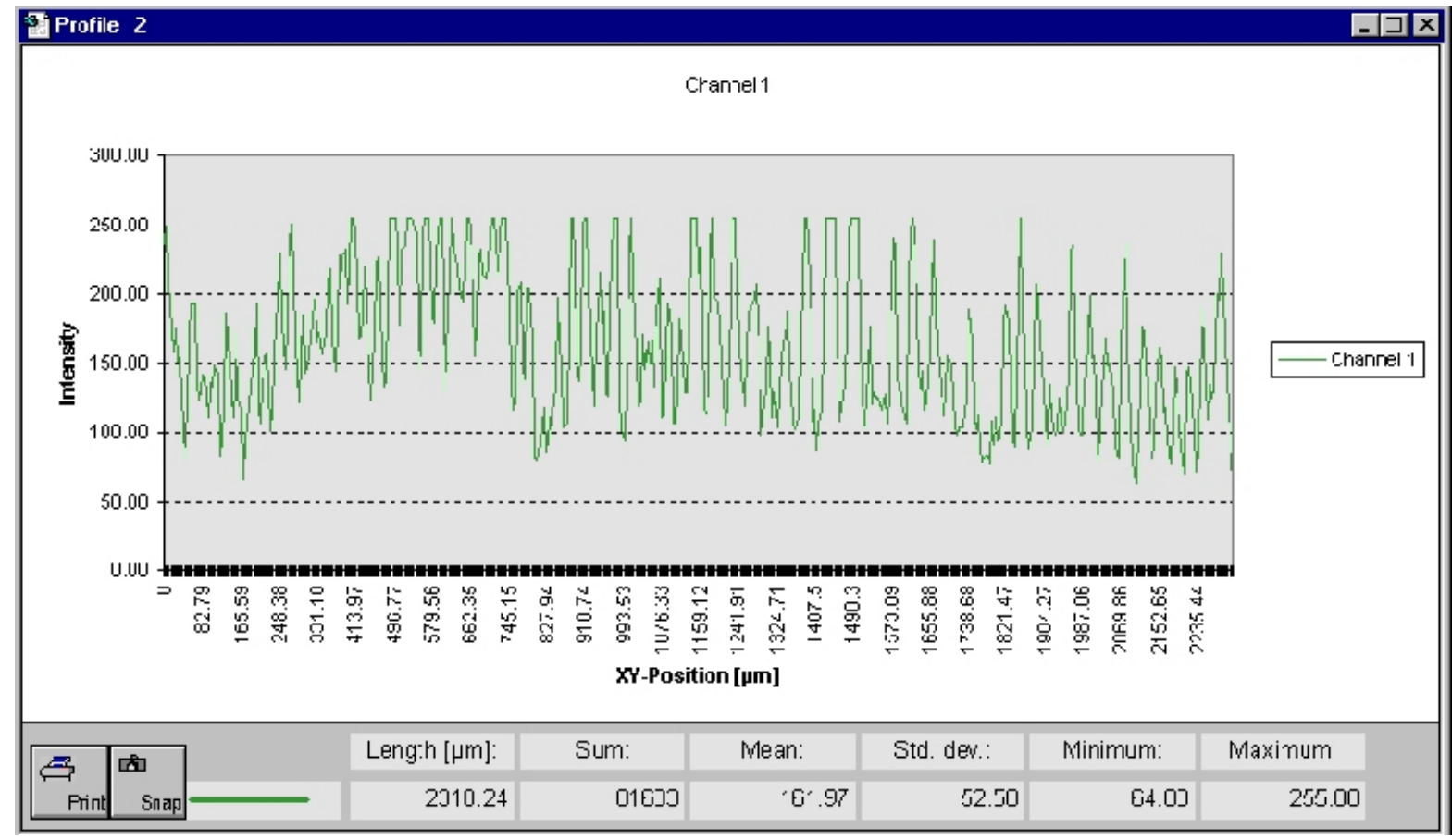

Figure 2. A quantitative software program measuring the intensity of the autofluorescence on a salmon scale. The profile suggests the age of the fish is 3 years old. 